\title{
Transit algorithm performance using real WASP data
}

\author{
B. Enoch ${ }^{1,2}$, C. A. Haswell ${ }^{2}$, A. J. Norton ${ }^{2}$, A. Collier-Cameron ${ }^{1}$, R. G. West ${ }^{3}$, A. M. S. Smith ${ }^{4}$, and N. R. Parley ${ }^{1,2}$ \\ 1 School of Physics and Astronomy, University of St. Andrews, North Haugh, St Andrews, KY16 9SS, UK \\ 2 Department of Physics and Astronomy, Open University, Walton Hall, Milton Keynes, MK7 6AA, UK \\ 3 Department of Physics and Astronomy, University of Leicester, Leicester, LE1 7RH, UK \\ 4 Astrophysics Group, Leonard-Jones Laboratories, Keele University, Staffordshire, ST5 5BG, UK
}

Received 7 June 2012 / Accepted 3 October 2012

\section{ABSTRACT}

\begin{abstract}
Context. Many transiting exoplanet surveys are now in operation, observing millions of stars and searching for the periodic signals that may indicate planets orbiting these objects.

Aims. We have tested the performance of transit detection algorithms using real WASP data, avoiding the issue of generating the appropriate level of white and red noise in simulated lightcurves. We used a two-dimensional search method, the box-least-squares (BLS) algorithm, and two- and three-dimensional versions of the analysis of variance (AoV) method.

Methods. After removing any potential transiting candidate or variable objects, transits were injected into each lightcurve. We performed Monte Carlo simulations, testing the recovery of injected signals in 99 lightcurves by each algorithm.

Results. In the simulations using data from one season of WASP observations, it was determined that the BLS method should detect a total of $37 \%$ of planets and the 3D AoV 23\%. Simulations to explore the effects of extending survey baseline or number of hours of observations per $24 \mathrm{~h}$ period, i.e. longitudinally spaced observatories, were also performed. They showed that increasing the coverage via an increase in baseline or in observational hours are equally good for maximising overall detections of transiting planets. The resulting algorithm performance was combined with actual WASP-South results to estimate that $0.08 \%$ and $0.30 \%$ of stars harbour very hot Jupiters and hot Jupiters respectively.
\end{abstract}

Key words. planets and satellites: detection - methods: data analysis - methods: statistical - planetary systems

\section{Introduction}

With the many transiting exoplanet surveys now in operation, there has been significant development of wide-field data reduction methods and transit detection algorithms. Unanticipated challenges in transit detection were encountered with regard to correlated (red) noise (Pont et al. 2006) in databases of lightcurves obtained.

Several types of transit-search algorithm exist, all of which aim to detect square-well shape transit events in stellar lightcurve data. All methods achieve this via a minimisation procedure, but the way in which parameter space is explored and the significance of a detection calculated both vary. Most groundbased, wide-field transit surveys that have confirmed several planet detections, including WASP (Pollacco et al. 2006), use variations of the method traditionally called box least squares (Kovács et al. 2002): see for example Bakos et al. (2007) for HAT, McCullough et al. (2005) for the XO project, and O'Donovan et al. (2006) for TrES. Alternative approaches include the matched filter algorithm (Jenkins et al. 1996; Weldrake $\&$ Sackett 2005), maximum likelihood method (Aigrain \& Irwin 2004; Burke et al. 2006) or hybrid reformulation of the BLS (Protopapas et al. 2005; Collier Cameron et al. 2006), waveletbased methods (Régulo et al. 2007; Carter \& Winn 2009) and the analysis of variance method described by SchwarzenbergCzerny \& Beaulieu (2006), also considered here.

Tingley (2003) and Moutou et al. (2005) investigated transit algorithm performance on ground-based and space-based synthetic data, respectively, where both synthesised white noise in their simulated lightcurves. Here, we use real WASP data to obtain results that are directly applicable to data of the quality and noise content actually seen in ground-based, wide-field surveys. The algorithms are applied to lightcurves detrended with the SysRem algorithm (Tamuz et al. 2005).

The BLS and AoV algorithms considered here are set out in Sect. 2, followed by the testing method in Sect. 3. The results are presented in Sects. 4-6 while Sect. 7 illustrates some weaknesses of algorithms. Section 8 then describes simulations exploring the effects on transit detection of various baselines and various numbers of hours of observations per 24-h period. Finally, Sect. 10 forms a discussion and conclusion of these results.

\section{Transit-search algorithms}

\subsection{Box least squares (BLS)}

The BLS algorithm used in these simulations is an implementation of the method of Kovács et al. (2002). This method searches a three-dimensional grid of frequency, box-width and phase, computing the signal residue (SR) value for each epoch at each frequency and width, using a box-shaped transit model and fitting for the best-fit depth (a single least-squares step and therefore not regarded as a dimension of the search). Given a lightcurve with $f_{i}$ representing the $i$ datapoints and $w_{i}=1 / \sigma_{i}^{2}$ being weights assigned to each datapoint, where $\sigma_{i}$ are the datapoint uncertainties, the SR is calculated by

$\mathrm{SR}=\frac{\left(\sum_{i \in t} w_{i} f_{i}\right)^{2}}{\sum_{i \in t} w_{i}\left[1-\sum_{i \in t} w_{i}\right]}$ 
where sums over $i \in t$ include only datapoints within the proposed transit. Once the SR has been calculated for each test frequency, the signal detection efficiency (SDE) is calculated from

$\mathrm{SDE}=\frac{\mathrm{SR}_{\mathrm{peak}}-\langle\mathrm{SR}\rangle}{s d(\mathrm{SR})}$

where $\langle\mathrm{SR}\rangle$ indicates the average value and $s d(\mathrm{SR})$ is the standard deviation of the SR values. Objects showing a strong SDE are selected as transit candidates.

\subsection{Analysis of variance (AoV)}

The algorithm used here is based on the analysis of variance (AoV) method described in Schwarzenberg-Czerny \& Beaulieu (2006) and summarised below. In the 3D implementation (period, duration, phase) for this work, a grid of frequencies, duration and bin-width are searched, where the frequency grid achieves Nyquist sampling such that over the entire data train of observations two consecutively tested frequencies cause the proposed event to move no more than half its duration. The duration grid searched consists of values where the longest duration, and thus lowest number of bins, corresponds to a central transit of a mid-F type star and the shortest duration, or highest number of bins, is that of a high-impact transit of a mid-K type star. A $2 \mathrm{D}$ version was also used, where the duration was kept fixed in the middle of this range.

The implementation gains its speed from the fast binning method described in Schwarzenberg-Czerny \& Beaulieu (2006), making use of sub-bins to reduce the number of calculations required. This results in an asymptotic speed of $\Theta\left(n^{0.23}\right)$, where $n$ is the number of datapoints in the lightcurve being analysed, resulting in the $\mathrm{AoV}$ implementation being fast to run on lightcurves with a large number of datapoints. Such a fast binning method can in fact be included in various transit search algorithm implementations; a similar method was put to use in the BLS implementation used here.

For each frequency and "binset" (duration or number of bins tested), the lowest, reasonably-sampled bin is selected as a possible transit event, provided that it is not unrealistically deep. Given flux values, $f_{i}$, flux uncertainties, $\sigma_{i}$, and corresponding weights, $w_{i}$, depth of possible event, $\delta$, and number of observations, $n$, the $\theta$ statistic is calculated from

$\theta(f)=\frac{R(f)}{(F-R(f))(n-2)}$

where

$F=\sum_{i} f_{i}^{2} w_{i}$

and

$R(f)=\frac{\delta^{2} \sum_{i \in t} w_{i} \sum_{i} w_{i}}{\sum_{i} w_{i}-\sum_{i \in t} w_{i}}$

sums over $i$ include all datapoints, sums over $i \in t$ include only datapoints within the proposed transit.

Over all frequencies, the highest $\theta$ value for each binset is $\mathrm{kept}$, and the significance of this maximum value is found by multiplying by the number of bins it was selected from. Over all binsets, the model with the best significance value is kept. In a dataset, objects with high significance values are determined to be good transit candidates by using the maximum observed $\theta$ value with the Fischer-Snedecor cumulative distribution to find the tail probability, $Q$, of the peak being significant.

We tested these algorithms in Monte Carlo simulations using real WASP lightcurves which were injected with transits, as described below. The threshold $\mathrm{SDE}$ and $Q$ significance values used in this work were chosen to be of practical use, regarding maximising the number of good candidates while keeping the number of lightcurves to be further investigated to a manageable level. The threshold values were chosen using a test field with good coverage from a single season's observations by one camera and set to select the top 5\% of candidates, ensuring that this easily contained the planet known to exist in the field.

\section{Comparison testing method}

Lightcurves using one season of data from a well-populated WASP-N field were selected in order to have a large number of objects with many datapoints. A well-covered field generally has data over a few months in any year, with around 30-50 datapoints per night. These lightcurves were filtered to include only those with a reasonable number of datapoints, around 5000, and brighter than $V=13$ mag. Using real lightcurves avoids the issue of making simulated lightcurves realistic, containing amounts of white (uncorrelated) and red (correlated) noise appropriate to such surveys.

To remove any lightcurves that showed candidate events, whether real or false positives, these objects were tested with each of the transit algorithms, and any reported candidates were removed. We also removed any objects that showed a high $\chi^{2}$ value when compared to a flat model, to remove other variable lightcurves such as those of deep eclipsing binaries, etc.

We randomly selected 99 lightcurves from those remaining, in percentage proportions for each half-magnitude bin between $V=9.5$ and 13.0 as represented in the entire WASP database, for example $25 \%$ of $9.5-13$ th magnitude stars within the WASP database have a $V$ magnitude between 12.0 and 12.5 so we chose 25 lightcurves to be in this magnitude range. This produced the final set of 99 lightcurves to be used in the transit detection tests.

At the beginning of each run, transits were injected into the randomly-reordered lightcurves of each of the final 99 objects. Periods of injected events ranged evenly from 1 to 5 days for lightcurves 1 to 99, in steps of 0.04 days. The stellar mass for each event was randomly selected to be between 0.7 and $1.3 M_{\odot}$, with the stellar radius then calculated as $R_{\text {star }}=M_{\text {star }}^{0.8}$. The planetary mass was selected within the range $0.4-2.0 M_{\mathrm{J}}$, with corresponding planetary radius based on a power law calculated from known transiting planets. The impact parameter was varied between 0 and 0.9. Event depths and durations were calculated based on selected stellar and planetary mass and radius, and impact parameter.

These transits were added to the lightcurves starting from a random orbital phase. Randomising the lightcurve order for each run ensured that lightcurves of different magnitudes and noise quality receive different period events each time transits are injected. Figure 1 provides examples of these lightcurves with transits injected, shown phase folded and with transits at 0.5 phase.

For each run, each algorithm was used to test the 99 injected lightcurves and the results were recorded before moving to the next run. 60 Monte Carlo runs, each testing the 99 lightcurves, were performed (simulation results converged to the final percentages after around 20-50 runs depending on the algorithm). Hence, each algorithm could potentially detect up to 99 simulated events per run, but noise and patchy coverage in the data 

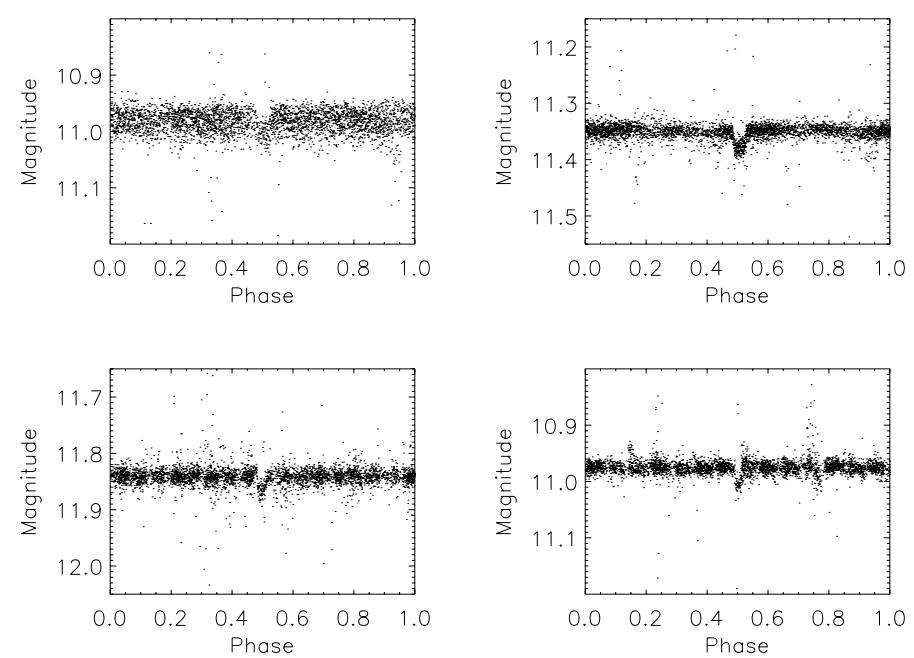

Fig. 1. Examples of lightcurves with transits. The injected events have periods of $1.97 \mathrm{~d}$ (top left), $2.05 \mathrm{~d}$ (top right), $4.27 \mathrm{~d}$ (bottom left) and $4.65 \mathrm{~d}$ (bottom right), all shown phase folded and with the transit at 0.5 phase.

Table 1. Overall detection results by algorithm for real data tests for runs of 99 objects.

\begin{tabular}{lcccc}
\hline \hline Algorithm & \multicolumn{2}{c}{ Detections/run } & $\sigma$ & S.E. \\
& (mean) & $\%$ & & \\
\hline 2D AoV & 15.33 & 15.49 & 2.68 & 0.35 \\
3D AoV & 22.90 & 23.13 & 2.95 & 0.38 \\
BLS & 36.63 & 37.00 & 3.52 & 0.45 \\
\hline
\end{tabular}

Notes. ( $\sigma$ and S.E. based on detection percentage values).

makes this highly unlikely. The total number of events each algorithm actually reported was recorded at the end of each run, and these totals were averaged at the end of all runs.

\section{Results of transit recovery}

The overall detection results are shown in Table 1, where $\sigma$ is the standard deviation of the number of detections, and S.E. is the standard error.

Thus, out of actual events existing in the WASP archive (if an equal number of events at all periods exist, but see Sect. 10 below), the $2 \mathrm{D} \mathrm{AoV}$ version should detect $15.5 \pm 0.7 \%$, the $3 \mathrm{D}$ AoV version should detect $23.1 \pm 0.8 \%$, and the BLS should detect $37.0 \pm 0.9 \%$ (using \pm 1.96 S.E. to give the $95 \%$ confidence interval). These results indicate that an effective algorithm should allow both period and duration to be fitted; the $2 \mathrm{D}$ version of AoV with duration held fixed performs significantly more poorly than the 3D version - see Sect. 7 for a discussion of this. The BLS method detects significantly more candidates.

Figure 2 shows the number of detections by each algorithm as a function of period, using the results collated over all runs. It shows which periods each algorithm is most and least efficient at detecting. All algorithms show a trend of more detections for shorter-period events than longer-period events, which is to be expected as shorter periods produce more events that will be sampled in the lightcurve for a given baseline. No spikes or dips in detections are seen at integer-day periods, as is to be expected for short period events - Gould et al. (2006) found only an insignificant 3\% enhancement in integer day resonances for 1-5 day transit survey detections.

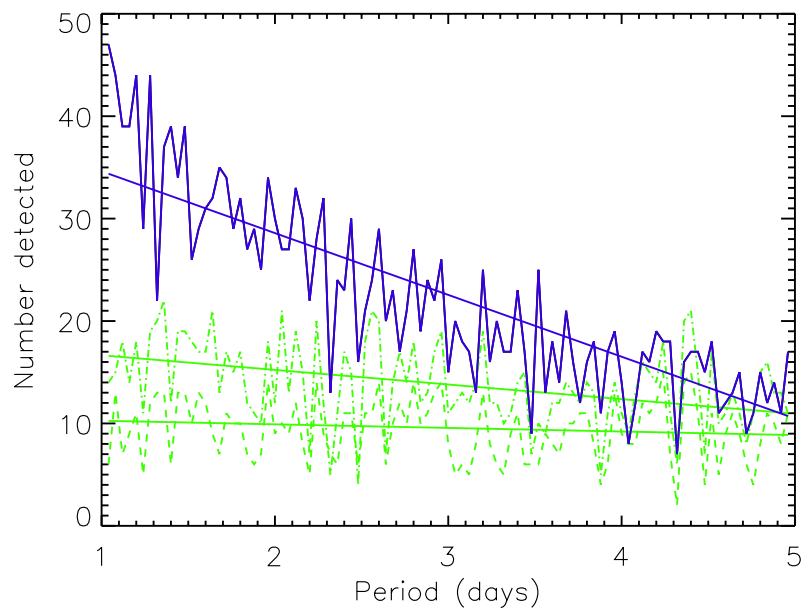

Fig. 2. Plot showing detection numbers versus period for each algorithm: blue/solid is BLS, green/dot-dashed is AoV 3D and green/dashed is AoV 2D. Best-fitting straight lines are over-plotted for each.

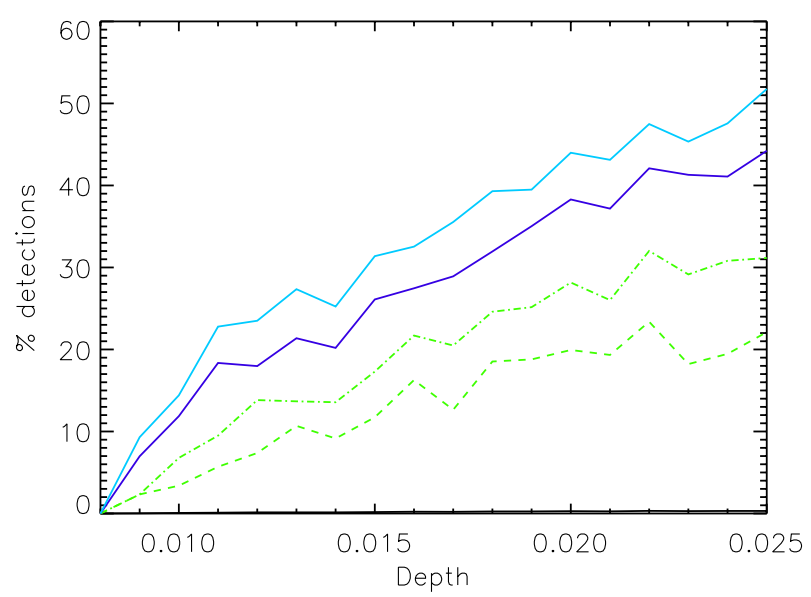

Fig. 3. Detections by algorithm varying by depth (mmag) of injected event: dark blue/solid is BLS, green/dot-dashed is AoV 3D, green/dashed in AoV 2D, and light blue/solid (top) is BLS+AoV-3D.

Examining the overlap of detections between the BLS and 3D AoV, we found that the overlap is not complete, meaning that the BLS detects objects that the AoV does not, and vice versa. This shows that each algorithm has certain strengths and weaknesses, and neither detects all events possible. The combined BLS and 3D AoV results are indicated on some of the graphs for illustrative purposes.

Further analyses of the detection results were performed to show the response of each algorithm to the event depth (Fig. 3) and to the magnitude of star the event was injected into (Fig. 4). As expected, all algorithms detect deeper events more easily. Perhaps slightly less expected is the result that each algorithm detects most events in stars of mid-range magnitude. This is likely to be because lightcurves of the brightest objects will be dominated by red-noise, producing artefacts that mimic or conceal transit events, whereas the dimmest objects will contain (and be dominated by) high levels of white noise, concealing any low-amplitude periodic signal. It is therefore easiest to detect transit events in mid-range magnitude objects in which white noise dominates over red noise but is not yet at sufficiently high levels to obscure the event signatures. 


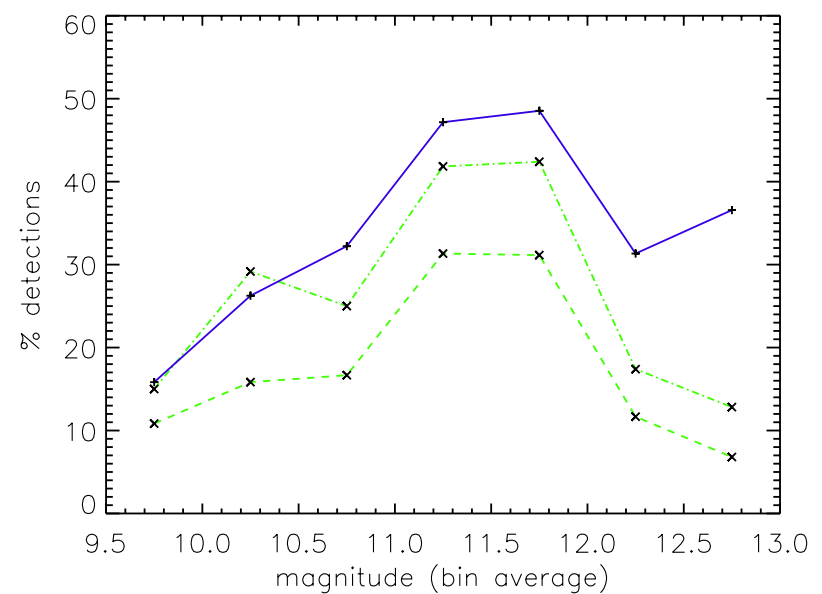

Fig. 4. Detections by algorithm varying by magnitude of star the event was injected into: dark blue/solid is BLS, green/dot-dashed is AoV 3D and green/dashed is AoV 2D. For example, the BLS algorithm detects almost $50 \%$ of events around 11-12th magnitude stars, but only $30 \%$ of transits around brighter 10.5 mag stars or dimmer 12.5 mag stars.

The results show that the BLS is much more efficient than the AoV in detecting short-period planets as well as those planets around fainter stars. This is due to the selection criteria: the BLS use of the SDE results in the selection of strong events relative to the rest of that object's periodogram and therefore will find strong peaks in a periodogram regardless of the absolute peak strength. In contrast, the AoV's use of the Fischer-Snedecor cumulative distribution to find the tail probability of significance of a peak will tend to detect peaks with strong absolute values even though the background level of the periodogram may be high. Thus, strong peaks against a low periodogram background level will tend to be detected by both BLS and AoV, strong peaks against a high periodogram background level will likely be detected by AoV but missed by BLS, and weaker absolutevalue peaks but against a low periodogram background level will likely be detected by BLS but missed by AoV. The second of those three possibilities is generally seen in stronger events in objects dominated by red noise, i.e. brighter stars with a high level of correlated noise. The third possibility is generally seen with weaker events around objects dominated by white noise, i.e. fainter stars. This suggests that BLS will detect more transiting planets around fainter (more numerous) stars than AoV, while AoV will detect some transiting events around brighter stars that BLS misses. This is what is seen in Fig. 4 and explains the result that BLS generally detects more planets than AoV while missing some events that AoV is capable of finding.

\section{Fraction of events detected above $S_{\text {red }}$ threshold}

In addition to calculating the recovery fraction of all the injected transit signals, it is useful to know the recovery fraction of the signals that are expected to produce a detectable signal-to-red noise, $S_{\text {red. }}$. Smith et al. (2006) calculate $S_{\text {red }}$ for a transit signal in a particular lightcurve using

$S_{\text {red }}=\frac{\delta \sqrt{N_{\mathrm{tr}}}}{\sigma_{\mathrm{r}}}$

where $\delta$ is the depth of the transit event, $N_{\text {tr }}$ is the number of transits that are visible in the lightcurve data and $\sigma_{\mathrm{r}}$ is the red noise of the lightcurve on timescales of order of a transit duration.

During each run then, each time a lightcurve was injected with an event, the number of visible injected transits was
Table 2. Detections by BLS and AoV algorithms for events above various $S_{\text {red }}$ thresholds.

\begin{tabular}{cccc}
\hline \hline$S_{\text {red }}$ & 3D AoV & BLS & Both \\
\hline 1.0 & 23.6 & 37.9 & 43.0 \\
2.0 & 26.4 & 42.4 & 48.1 \\
3.0 & 32.3 & 52.5 & 58.8 \\
4.0 & 38.7 & 65.5 & 71.4 \\
5.0 & 43.9 & 74.7 & 80.1 \\
\hline
\end{tabular}

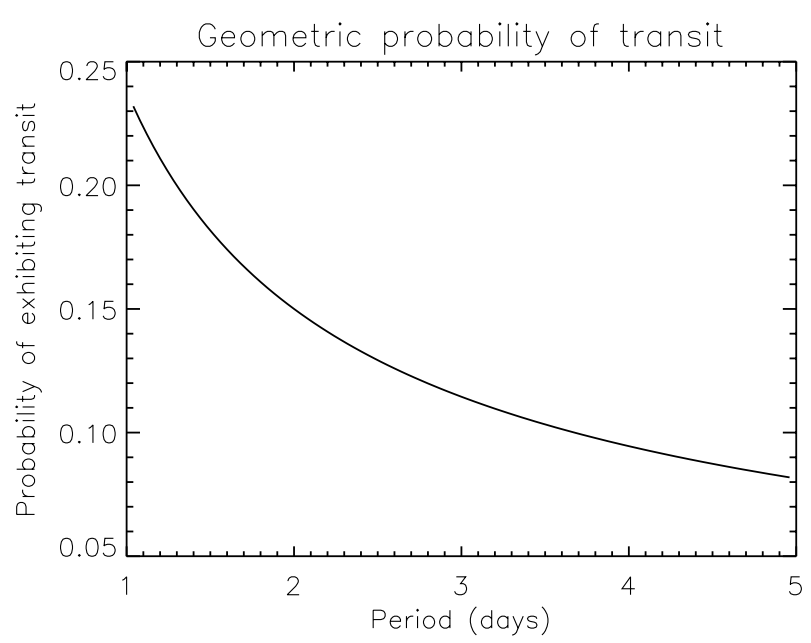

Fig. 5. Plot showing probability of planets being in transiting inclination for systems with periods between 1 and 5 days.

recorded, and the level of red noise of the lightcurve was calculated based on the rms of a sliding average $2.5 \mathrm{~h}$ bin (the approximate timescale of a transit). Table 2 shows the percentages of events detected above certain signal to red noise, $S_{\text {red }}$, thresholds for the AoV, BLS, and both combined.

\section{Selection effects influencing overall planet yield}

Assuming that hot Jupiter planets show no preference for a particular semi-major axis, $a$, the distribution of planets is flat with respect to $a$. However, planets are geometrically more likely to transit their host star the closer they are to it, which causes the distribution of planets visible by transit to peak towards lower values of $a$. This is due to simple geometry: the probability of a planet showing a transit is given by

$p=\frac{R_{*}}{a}$

assuming that $R_{\mathrm{p}} \ll R_{*}$ and a circular orbit (Sackett 1999).

To account for this variation by period of the detectability of planets by transit methods, we calculated the probability of transit at each period of injected event, between 1.0 and 5.0 days. The results are shown in Fig. 5, showing that for example a planet in an orbit around a $1 R_{\odot}, 1 M_{\odot}$ star with period 1 day is about three times more likely to show a transit than a planet in an orbit with period 5 days, purely due to the geometry of the systems.

If exoplanets are, during formation, not biased towards having any particular final semi-major axis (though this may not be true, see Sect. 10 below), Fig. 5 shows that more short-period transiting hot Jupiters should be detected than longer-period hot Jupiters since more of them are visible to us. 


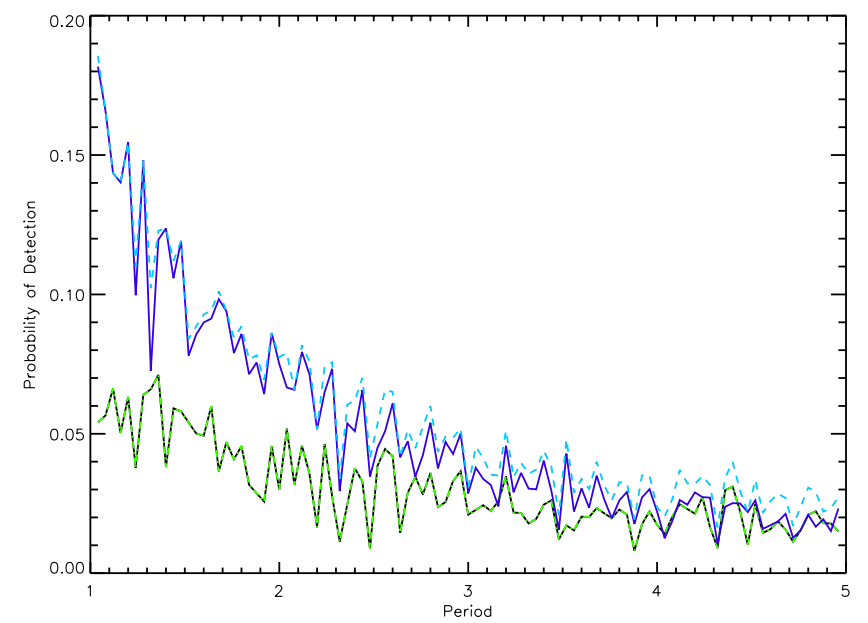

Fig. 6. Plot showing actual detection distribution multiplied by the probability of a transiting inclination. Dark blue/solid is BLS, greenblack/dot-dashed is the $3 \mathrm{D} \mathrm{AoV}$ and the light blue/dashed is both combined.

Therefore, to convert the algorithm performance results from the above simulations into a conclusion about expected levels of hot Jupiter transit events detections in actual WASP datasets, the period detection distribution of each algorithm determined from the simulation results was multiplied by the geometric probability of a transiting inclination. This resulted in Fig. 6. Assuming that an equal number of planets exist around well-sampled stars at each tested period somewhere in the WASP dataset, by summing over all periods it is found that the BLS would detect a total of $41.3 \%$ of potentially detectable (i.e. transiting) planets, the $3 \mathrm{D}$ AoV would detect $23.9 \%$, and both combined should detect $45.6 \%$. The slightly higher percentage values compared to the direct results from the simulations are due to both curves peaking towards shorter periods.

\section{Algorithm weaknesses}

A two-dimensional approach, such as the 2D AoV considered here, or the 2D hybrid BLS algorithm of Collier Cameron et al. (2006) based on Protopapas et al. (2005) and similar to the reformulation developed by Aigrain \& Irwin (2004) and Burke et al. (2006), keeping the box width fixed and fitting for best-fit depth, may not maximise detections. Keeping the box width fixed allows a "standard" transit event, such as that of WASP-1 (see Fig. 7), to be well-detected. However, events that are longer or shorter than this, e.g. due to a smaller star, high impact parameter or both, such as that of WASP-2, will be recorded at lower significance than had the box width been more optimally fitted. This is due to some out-of-transit datapoints being included in the box width, reducing the fitted depth of the event and therefore reducing the significance of the detection.

The varying $\Delta \chi^{2}$ relative to a flat-line fit from a flat distribution of transit models with various event durations is shown in Fig. 8 for the transit lightcurve of WASP-2. Clearly, the 2D algorithm, fitting for a longer duration, does not achieve the maximum possible significance for the event. This causes shortduration, shallow events such as a high-impact parameter transit of a late-type star, to be missed as they will be recorded at too low a significance to be detected.

Effective algorithms should therefore not ignore the important variable of duration of transit, but should allow this to vary.

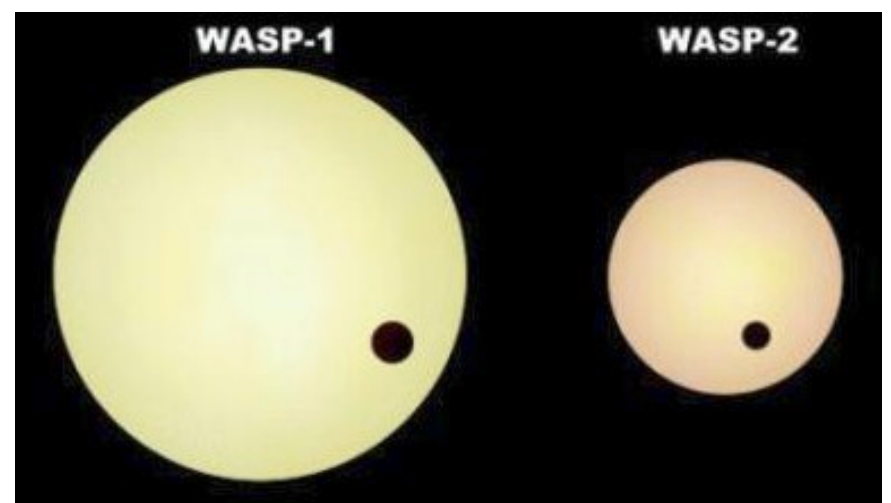

Fig. 7. Shows the differences between the WASP-1 and WASP-2 systems: WASP-1b transits an F7V star with an impact parameter close to 0; WASP-2b transits a much smaller, K1V star, with a high impact parameter. This results in a shorter, deeper transit for WASP-2b compared to that of WASP- $1 \mathrm{~b}$.

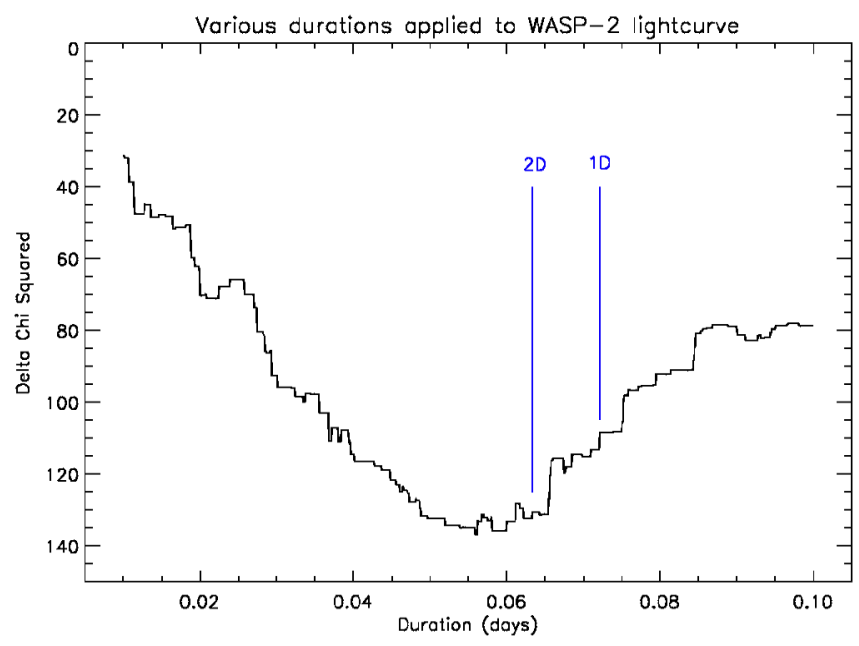

Fig. 8. Plot showing the $\Delta \chi^{2}$ from a flat distribution of various transit durations, with depth optimally fitted for each duration. The $2 \mathrm{D}$ algorithm results in a longer duration, and therefore less significant event, than the 3D approaches which vary the durations searched over.

\section{Multi-baseline and location}

Further simulations were performed to investigate the effect of survey baseline and hours of coverage per 24-h period on the detection rate for each algorithm. Three sets were run with different baselines - around 1.5, 3 and 6 months - each with subsets for 4, $8,12,16,20$ and $24 \mathrm{~h}$ of observations per $24 \mathrm{~h}$ period, simulating the combination of data from several telescopes spaced in longitude. The same real WASP lightcurves as above were used, resampled in random $2.5 \mathrm{~h}$ intervals to fill in nightly coverage and extend the baseline (though not accounting for airmass dependence of the red-noise). This provided realistic lightcurves at the required baselines and coverages, retaining the noise profile of the original WASP lightcurves and containing gaps due to poor weather.

Once each set of lightcurves was prepared, the simulations were carried out in the same way as above, with transit events being injected into each lightcurve and run through each algorithm. 50 runs were performed on each set, and detections by each algorithm were recorded. The results are shown in Fig. 9.

As could be expected, detections rise for all algorithms as the number of hours of observation per $24 \mathrm{~h}$ period rises. Detections also rise as the survey baseline increases, since the significance 


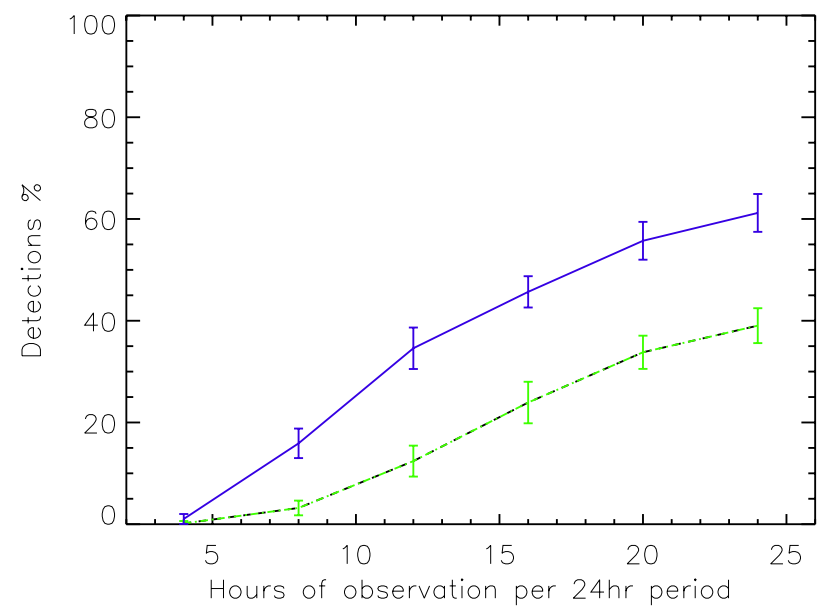

(a) 1.5 month baseline

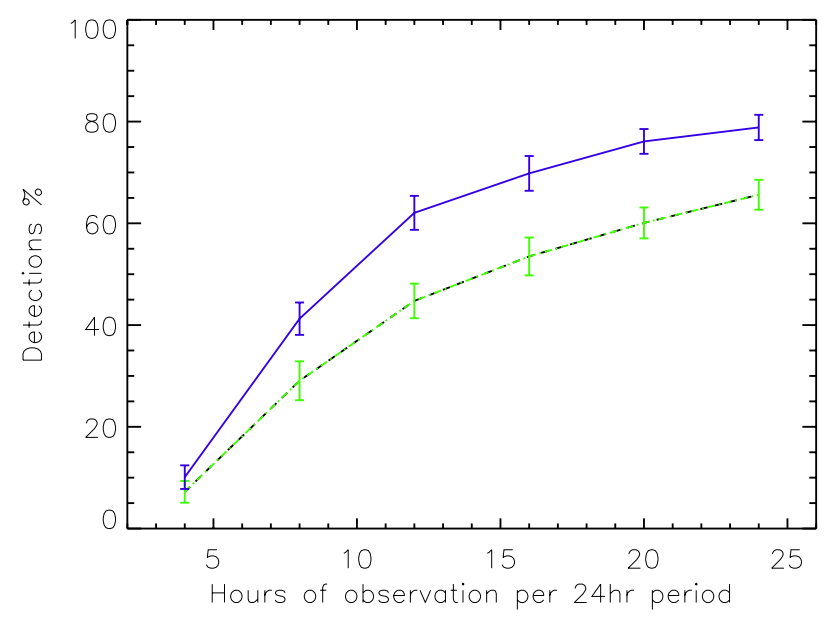

(b) 3 month baseline

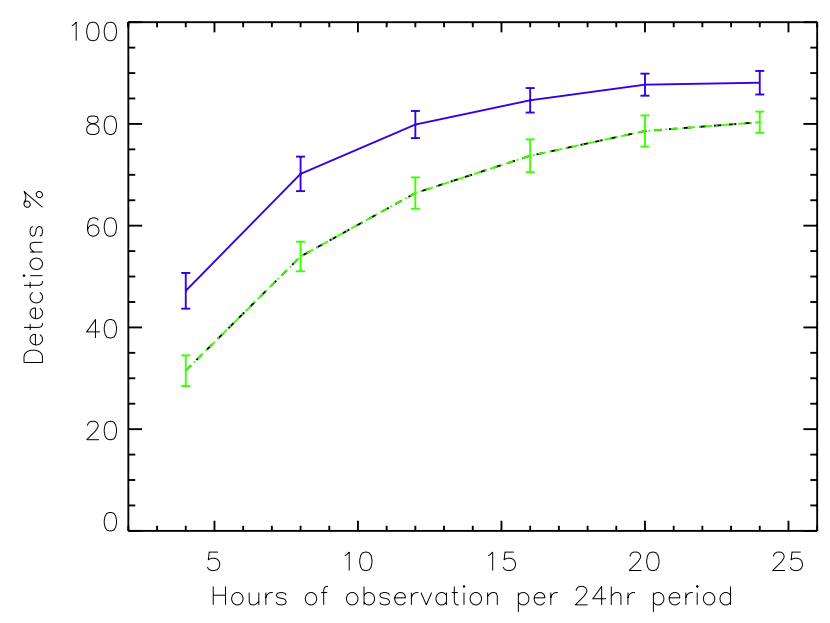

(c) 6 month baseline

Fig. 9. Shows the effect of varying the number of hours of observation per night for various baselines of observations. Dark blue/solid is BLS, green/dot-dashed is the $3 \mathrm{D} \mathrm{AoV}$ and green/dashed is the $2 \mathrm{D} \mathrm{AoV}$.

of detections generally rises as more transit events may be observed.

By comparing the results from each baseline it can be shown that a similar percentage of detections can be obtained from a survey over six months with one camera (around $6 \mathrm{~h}$ of observations per night) as a three month survey using two cameras spaced in longitude that could provide $12 \mathrm{~h}$ of observations per $24 \mathrm{~h}$ ( $\sim 45 \%$ for AoV and $\sim 60 \%$ for BLS). This shows that increasing the coverage via an increase in baseline or in observational hours are equally good for maximising overall detections of transiting planets. Further examples are seen in eg the increase in detections by the BLS from $40 \%$ with $8 \mathrm{~h}$ per day observations over 3 months to $70 \%$ either by doubling the baseline to 6 months ( $8 \mathrm{~h}$ per day) or doubling the observational hours to $16 \mathrm{~h}$ per day (3 month baseline).

The long-baseline results show the BLS algorithm leveling off at almost $90 \%$ detections with $24 \mathrm{~h}$ a day coverage, indicating that all hot Jupiter events are unlikely to ever be detected in a transit survey - some are too shallow to see among the noise.

\section{Application to WASP results}

Around 200 transiting planets have now been confirmed (www. exoplanet.eu) through a variety of wide-field or targeted, shallow or deep surveys. Most wide-field, shallow searches operate(d) in the Northern hemisphere, including WASP, HAT, $\mathrm{XO}$ and TrES but in the Southern hemisphere WASP has until recently been the only survey searching for transits around bright stars; planets in the Southern hemisphere found by OGLE, SWEEPS and CoRoT are around stars fainter than $V=13 \mathrm{mag}$, down to around $18 \mathrm{mag}$. The wide-field survey HAT-South has only very recently (June 2012) announced its first planet detection (Penev et al. 2012). The results from WASP-South may therefore be used together with statistics on stars observed and transit algorithm performance to estimate the number of stars harbouring very hot Jupiters (1-3 day orbital period) or hot Jupiters (3-5 days).

WASP-South has found 39 planets with 1-5 day periods around stars of 9.5-13 mag stars. Table 3 shows numbers of stars in this magnitude range observed by WASP-South, along with number of datapoints. Here, stars with 1000-3000 points are considered to have been observed for one half a "standard" baseline as set out in the above simulations, stars with 30006000 have one standard baseline (around 3 months or 4000 datapoints), 6000-10000 have two, etc. up to stars with above 30000 having at least eight standard baselines of observations.

In Sect. 4 we showed that it is unimportant for overall detections whether the observations are carried out quickly and intensively (e.g. $24 \mathrm{~h}$ a day monitoring for a few months) or sparsely over a long temporal baseline (a few hours a day over many months). We estimate the percentage detections expected for each of 0.5 to 8 baselines of observations: detections for half, one and two baselines at $6 \mathrm{~h} /$ day observations are read directly from Figs. 9a to c, three baselines is the equivalent of $18 \mathrm{~h}$ /day from Fig. 9b, four is the equivalent of $12 \mathrm{~h}$ /day from Fig. 9c, etc. At eight baselines, e.g. 24 h/day in Fig. 9c the levelling off at around $88 \%$ detections is reached, therefore all stars with over 30000 datapoints are considered to have had $88 \%$ of transiting hot and very hot jupiters detected.

Table 3 begins with numbers of stars per observation length range, then multiplies this by $10 \%$ to roughly obtain the approximate number of stars for which WASP will be sensitive to transiting hot Jupiters, avoiding stars that are too large (early-type stars and giants) to allow detection of planets (Gould \& Morgan 2003). Next, this is multiplied by $10 \%$ or $15 \%$ for HJ and VHJ respectively to find the likely number of planet-star systems with the correct geometry to be observed via transits (Fig. 5). Finally the transit search algorithm performance percentage is applied to determine the number of stars that should have had transits detected, if all stars harboured hot or very hot Jupiters. 
B. Enoch et al.: Transit algorithm performance using real WASP data

Table 3. Stars observed by WASP-South with number of datapoints, adjusted for suitability, star-planet geometry, and detection percentages.

\begin{tabular}{|c|c|c|c|c|c|c|}
\hline Points $(, 000)$ & Num stars & $\times 10 \%$ suitability & Algorithm performance & Performance adjusted & $\times 15 \% \mathrm{VHJ}$ & $\times 10 \% \mathrm{HJ}$ \\
\hline $1-3$ & 100872 & 10087 & 0.10 & 1009 & 151 & 101 \\
\hline $3-6$ & 124261 & 12426 & 0.25 & 3107 & 466 & 311 \\
\hline $6-10$ & 168042 & 16804 & 0.55 & 9242 & 1386 & 924 \\
\hline $10-14$ & 223874 & 22387 & 0.77 & 17238 & 2586 & 1724 \\
\hline $14-18$ & 184081 & 18408 & 0.80 & 14726 & 2209 & 1473 \\
\hline $18-22$ & 185092 & 18509 & 0.83 & 15362 & 2304 & 1562 \\
\hline $22-26$ & 134136 & 13414 & 0.85 & 11402 & 1710 & 1140 \\
\hline $26-30$ & 73518 & 7352 & 0.87 & 6396 & 959 & 637 \\
\hline Total & & & & & 13711 & 9380 \\
\hline
\end{tabular}

By comparing the result from Table 3 that 13711 and 9380 stars could potentially have had VHJ and HJ detected, respectively, with the actual number of planets discovered by WASP-South - $11 \mathrm{VHJ}$ and $28 \mathrm{HJ}$ - it can be estimated that $0.08 \%$ of stars have very hot Jupiters and $0.30 \%$ of stars have hot Jupiters orbiting them. Gould et al. (2006) made a similar estimate using OGLE data, finding $0.15 \%$ and $0.26 \%$ of stars have VHJ and HJ orbiting, respectively, though this was based on a small number of detected planets and their estimate did not account for algorithm detection performance.

\section{Conclusion}

We have shown that three-dimensional algorithms (searching period/frequency and duration space) perform better than twodimensional approaches. Of the 3D methods tested here, the BLS algorithm performs better than the AoV algorithm: the SDE selection criteria of signal detection efficiency seems more robust than the tail-probability selection criteria of the AoV algorithm.

We have used real WASP lightcurves to determine the transit algorithm performance by number of observations of a star, finding a maximum of around $90 \%$ detections for a set of stars with 30000 datapoints. We have used the algorithm performance results to estimate that $0.08 \%$ and $0.30 \%$ of stars harbour VHJ and $\mathrm{HJ}$ planets, respectively.

The larger percentage of stars with HJ compared to those with VHJ (along with the actual numbers of these detected by WASP-South) suggests the final orbital distance after giant planet migration towards its host star is not flat. Fewer VHJ are observed than $\mathrm{HJ}$; the opposite is expected from simple geometrical considerations alone. This implies that either very short period orbits are unstable, or there is a stopping mechanism that halts migration of giant planets before this, at around the 3 day orbital period mark, where there is a pile-up in detected planets (see for example exoplanet.eu, Udry \& Santos 2007; or Jackson et al. 2009). We are beginning to quantify the dependence of planeticity on orbital characteristics, but there remains much work to be done.

Further analyses could be performed in a similar manner to extract the dependence of planeticity on stellar spectral type and other parameters, particularly after GAIA provides information on all the bright stars of the Galaxy.

Acknowledgements. WASP-North is hosted by the Isaac Newton Group on La Palma and WASP-South is hosted by the South African Astronomical Observatory (SAAO) and we are grateful for their ongoing support and assistance. Funding for WASP comes from consortium universities and from the UK's Science and Technology Facilities Council.

\section{References}

Aigrain, S., \& Irwin, M. 2004, MNRAS, 350, 331

Bakos, G. Á., Noyes, R. W., Kovács, G., et al. 2007, ApJ, 656, 552

Burke, C. J., Gaudi, B. S., DePoy, D. L., \& Pogge, R. W. 2006, AJ, 132, 210

Carter, J. A., \& Winn, J. N. 2009, ApJ, 704, 51

Collier Cameron, A., Pollacco, D., Street, R. A., et al. 2006, MNRAS, 373, 799

Gould, A., \& Morgan, C. W. 2003, ApJ, 585, 1056

Gould, A., Dorsher, S., Gaudi, B. S., \& Udalski, A. 2006, Acta Astron., 56, 1

Jackson, B., Barnes, R., \& Greenberg, R. 2009, ApJ, 698, 1357

Jenkins, J. M., Doyle, L. R., \& Cullers, D. K. 1996, Icarus, 119, 244

Kovács, G., Zucker, S., \& Mazeh, T. 2002, A\&A, 391, 369

McCullough, P. R., Stys, J. E., Valenti, J. A., et al. 2005, PASP, 117, 783

Moutou, C., Pont, F., Barge, P., et al. 2005, A\&A, 437, 355

O’Donovan, F. T., Charbonneau, D., Torres, G., et al. 2006, ApJ, 644, 1237

Penev, K., Bakos, G. Á., Bayliss, D., et al. 2012, AJ, submitted [arXiv: 1206. 1524]

Pollacco, D. L., Skillen, I., Cameron, A. C., et al. 2006, PASP, 118, 1407

Pont, F., Zucker, S., \& Queloz, D. 2006, MNRAS, 373, 231

Protopapas, P., Jimenez, R., \& Alcock, C. 2005, MNRAS, 362, 460

Régulo, C., Almenara, J. M., Alonso, R., Deeg, H., \& Roca Cortés, T. 2007, A\&A, 467, 1345

Sackett, P. D. 1999, in NATO ASIC Proc. 532: Planets Outside the Solar System: Theory and Observations, eds. J.-M. Mariotti, \& D. Alloin, 189

Schwarzenberg-Czerny, A., \& Beaulieu, J.-P. 2006, MNRAS, 365, 165

Smith, A. M. S., Collier Cameron, A., Christian, D. J., et al. 2006, MNRAS, 373, 1151

Tamuz, O., Mazeh, T., \& Zucker, S. 2005, MNRAS, 356, 1466

Tingley, B. 2003, A\&A, 408, L5

Udry, S., \& Santos, N. C. 2007, ARA\&A, 45, 397

Weldrake, D. T. F., \& Sackett, P. D. 2005, ApJ, 620, 1033 